\title{
Plantes transgéniques, expertise et action publique : évolution de la place et du rôle de la CGB de 1986 à $2006^{1}$
}

\author{
Christophe BONNEUIL ${ }^{1}$ \\ Pierre-Benoît JOLY² \\ ${ }^{1}$ CNRS, Centre Koyré d'histoire des sciences et \\ des techniques, MNHN, CP 25, 57 rue Cuvier, \\ 75231 Paris cedex 05 \\ <bonneuil@damesme.cnrs.fr> \\ ${ }^{2}$ Unité TSV, INRA
}

Nous porterons un regard d'analystes des sciences sociales sur la manière dont la Commission du génie biomoléculaire (CGB) a traversé vingt ans de transformation de l'expertise en France, de transformation de l'espace public et du débat sur les OGM, et vingt ans d'inflexion de sa place dans le dispositif d'action publique sur les plantes transgéniques. Nous nous appuyons sur nos recherches et sur plusieurs autres travaux de sciences sociales auscultant la fabrique de l'expertise à la CGB [1-5]. Nous verrons que le cas de la CGB illustre des transformations beaucoup plus larges entre science et société.

\section{Des périodes caractéristiques}

Les vingt années d'existence de la CGB nous semblent pouvoir être caractérisées autour de cinq périodes :

- 1986-1992.

La création d'un comité en 1986-1987 auprès d'une direction ministérielle, à représentation ex nomine d'entreprises, de syndicats et d'agriculteurs. À cette époque la France est le principal guichet d'entrée des OGM en Europe, et la contestation publique est très faible dans I'Hexagone, à la différence des États-Unis, de I'Allemagne ou de l'Europe du Nord. II s'agit alors pour les responsables, en quelque sorte, d'exploiter cet "avantage comparatif». La gouvernance du dossier OGM est, à l'époque, largement protégée d'un débat public peu présent.

\footnotetext{
1 Texte de la conférence prononcée dans le cadre de la journée «Les 20 ans de la Commission du Génie Biomoléculaire », le 20 septembre 2006. Actes disponibles: (http://www.ogm.gouv.fr/experimentations/ evaluation_scientifique/cgb/CGB.htm).
}

\begin{abstract}
Through the history of the Commission du Génie Biomoléculaire, we analyse the transformations of the expertise and regulation of GM crops in France since 1986. These transformations illustrate wider changes in the relations between scientific advice, policy, research communities and public arenas (media, courts...).
\end{abstract}

Key words: GM crops, genetic engineering, advisory committees, controversy, history of science and technology, sociology of science and technology

- 1992-1996.

Dans une seconde période le Comité est peu modifié dans sa composition et ses pratiques, mais il est recréé par la loi de 1992 qui transpose la directive européenne de 1990. Fait intéressant, avant la controverse publique, les premières divergences $s^{\prime}$ expriment entre les experts.

\section{- 1996-1998.}

Entre mi-1996 et mi-1998, la CGB entre dans le tourbillon de la controverse publique. En 1998, la CGB est alors renouvelée pour mettre en œuvre les principes de transparence et d'expertise contradictoire.

\section{- 1998-2003.}

Mais l'activité de la CGB reste ralentie faute de dossiers en contexte de moratoire européen puis de fauchages d'essais, et s'exerce dans un paysage plus large et plus diversifié de l'expertise (apparition de nouvelles instances et du modèle des agences : comité OGM de l'Afssa ; évolution du dispositif européen et rôle de I'Efsa). On note aussi une contestation systématique des avis dans l'arène judiciaire.

\section{- 2003-2006.}

Tandis que le nombre de dossiers traités par la CGB augmente de nouveau peu à peu, la levée du moratoire européen en 2003 ouvre une nouvelle phase pour la Commission bien que le Gouvernement connaisse de grosses difficultés à clore la controverse et ouvrir la porte aux PGM dans un contexte de fortes mobilisations citoyennes.

Nous parlerons essentiellement des 4 premières phases, en laissant le soin aux protagonistes d'interpréter eux-mêmes la phase en cours !

\section{Les grands traits des dix premières années}

Nous brosserons les grands traits des deux premières périodes tant sous l'angle des rapports entre sciences, expertise et décision, que de la philosophie, de la rigueur et des niveaux d'exigence du travail d'expertise de la Commission.

\section{Recherche, expertise et décision : trois fonctions superposées}

Par contraste aux dernières années, la relation entre recherche, expertise et décision paraît très fusionnelle dans la première décennie de la CGB.

Il existe une intrication entre recherche et travail d'expertise. D'abord, parce que ce sont les premières années de la transgenèse (la première plante transgénique fut obtenue en laboratoire en 1983) et qu'on connaît encore mal les propriétés et les impacts des nouvelles entités « chimères » qui sortent des laboratoires. Les incertitudes sont alors nombreuses. Le travail de la $C G B$, réellement exploratoire, présente un fort contenu cognitif. II ne s'agit pas d'opérer une simple synthèse des connaissances existantes comme dans certaines autres configurations d'expertise, mais bien de produire de la connaissance. Le lien entre expertise et front de la connaissance est par conséquent très étroit.

D'autre part, on se situe alors dans un contexte de genèse d'une technologie, au sein d'un petit monde de pionniers. Les experts internes et externes des 5 ou 6 premières années sont eux-mêmes des protagonistes de la transgenèse végétale qui s'évaluent les uns les autres, écrivent des rapports sur les enjeux des biotechnologies pour l'Office parlementaire des 
choix scientifiques et techniques et participent aux cercles nationaux ou européens où se définissent les priorités de recherche en biotechnologie [6].

Enfin, le lien est très fort avec la recherche puisque ce sont les membres de la CGB qui sont à l'origine des quelques appels de recherche sur les risques potentiels des OGM et qui « cadrent » les risques qui leurs semblent mériter investigation ${ }^{2}$.

À cette époque-là, l'expertise est tout aussi fusionnelle avec la décision. La séparation entre l'évaluation et la gestion des risques est une distinction que l'on ne verra émerger que plus tard. Les avis de la CGB sont alors explicitement formulés comme "favorables » ou " défavorables », contrairement aux formulations actuelles qui sont plus distantes et ménagent un espace à la décision politique. Pendant les 10 premières années, ces avis sont toujours suivis d'une décision conforme du ministère et sont même, jusqu'en 1992, transmis directement aux pétitionnaires sous la signature du président. La Commission réalise là un travail d'évaluation qui est très proche de la décision, comme le montre par exemple cet extrait d'un avis du 20/5/91 (dossier 91.03.02) : " la CGB (...) indique que les expérimentations décrites ne présentent pas de danger a priori et peuvent donc être entreprises. "Ce type de formulation a disparu des avis récents.

\section{Accompagner l'innovation}

Comme Gilles Pelsy l'a noté et comme Alexis Roy l'avait montré, la CGB se donne alors pour mission d'accompagner l'innovation sans entraver. Ni le feu vert de l'administration ni même la déclaration des essais d'OGM n'est obligatoire avant les décrets d'application de la loi de juillet 1992. Le dispositif repose sur le volontariat des pétitionnaires et, dans ce contexte, la CGB conçoit son rôle comme une démarche de "pédagogie » en direction des entreprises et des chercheurs, et non pas comme « couperet réglementaire ». En outre, on se trouve alors entre personnes compétentes et soucieuses de la compétitivité des biotechnologies françaises. Cet objectif supérieur de développement des biotechnologies est partagé par les membres de la commission et, dans ce contexte, il ne viendrait à personne l'idée d'étanchéifier la frontière entre activité de la CGB et monde des entreprises biotechnologiques. "Chimie et Ecologie », organisation de lobbying des industries chimiques et biotechnologiques, est alors un partenaire naturel qui coproduit les lignes directrices en matière

\footnotetext{
${ }^{2}$ Ce n'est qu'à partir de 1998 que ce domaine de recherche va s'autonomiser de la CGB avec des appels, aux «cadrages » multiples, de I'Inra, du CNRS et du ministère de la Recherche.
}

de sécurité, inspire des démarches de la CGB auprès des députés européens afin d'adoucir la directive 90-220. C'est aussi à « Chimie et Ecologie », qu'est confié un des premiers projets de recherche sur les risques des OGM.

\section{Un cadrage des risques inscrit dans le paradigme de la biologie moléculaire}

Comme nous I'avons indiqué, on avait alors affaire à de nouveaux objets sortis des laboratoires en 1983. II s'agissait donc d'explorer et de qualifier la nature de ces objets, leurs propriétés, leurs risques potentiels. Ce travail $d^{\prime}$ appréhension scientifique des risques est, dans la première décennie de la CGB, essentiellement cadré ${ }^{3}$ selon les lorgnettes de la biologie moléculaire, paradigme dominant la biologie française dans les années 1980. "On était des biologistes moléculaires, se souvient ainsi Axel Kahn, et donc on avait bien précisé quel était notre rôle. (...) Et les cartésiens que nous étions partaient du principe que pour se prononcer sur l'absence de risque, il fallait connaître les événements. (...) on ne peut pas, d'une certaine manière, se prononcer sur le risque d'un événement pas connu (...) on a introduit l'exigence d'une description parfaite de l'évènement moléculaire, et ça c'était complètement nouveau. » (A. Kahn, entretien du 5/9/06). Pour savoir ce qu'il fallait tester, il s'agissait donc en premier lieu de déterminer la nature moléculaire de ces événements de transformation. Le risque est alors vu comme une fonction de la " propreté » de la construction et quelque chose que l'on peut estimer en additionnant les caractéristiques de la plante hôte et les caractéristiques des transgènes introduits. Dans un paradigme où I'ADN est considéré comme une molécule programme stable, et c'est là l'héritage du dogme central de la biologie moléculaire des années 1960, les OGM apparaissent comme plus prédictibles et plus sûrs que les variétés classiques [7].

Si l'approche moléculaire est dominante, des questionnements plus agronomiques sur les OGM se font jour assez tôt également lorsque la CGB se déclare "assez réticente et inquiète face à un développement inconsidéré de la technique consistant à transférer dans des plantes des gènes de résistance à des herbicides. Une généralisation de ces techniques pourrait aller dans le sens d'une utilisation très large des herbicides, d'une diminution de la diversité génétique, et de

\footnotetext{
${ }^{3}$ La notion sociologique de cadrage $d^{\prime}$ un problème (frame) se comprend aisément par la métaphore photographique : ce qui est dans le champ ou non (les questions mise en avant ou non), ce qui est net ou non (au sein des questions mises en avant, certaines sont considérées comme présentant des incertitudes méritant recherche tandis que d'autres sont considérées comme résolues).
}

problèmes difficiles auxquels serait confronté l'agriculteur lorsqu'existeront, dans les cultures, des plantes d'autres espèces, résistantes à un herbicide, échappées de cultures voisines » [8].

\section{Des exigences fortes sur les constructions}

La feuille de route que se donne initialement la CGB, sous l'impulsion d'Axel Kahn qui la préside de 1988 à 1997, c'est le « risque zéro », car en matière alimentaire, "à la différence du secteur médical, le droit au risque n'y est pas admis. Par ailleurs, un certain attachement à la tradition (...) trouble la perception que le public peut avoir du progrès technique. De ce fait le progrès technique doit être conduit avec doigté afin d'éviter la survenue de sentiments irrationnels » [9].

Pour assurer la sécurité des OGM sans pour autant alerter les consommateurs, le paradigme de la biologie moléculaire semble offrir la solution : des constructions génétiques qui soient « propres », « la modification génétique limitée à l'essentiel descriptible et reproductible » [8]. Sur ce critère moléculaire, la CGB est au début des années 1990 la commission la plus exigeante des pays industriels. Ainsi les exigences des experts français, dans les discussions de l'OCDE en 1991, sont-elles supérieures à celles de leurs collègues anglais ou américains. Dans cette perspective également on note un rejet initial des gènes marqueurs de résistance aux antibiotiques. Plusieurs avis reprennent le type de préconisations suivantes : " $L a$ CGB suggère que les constructions soient simplifiées, et que, au moins pour les prochaines constructions, un effort soit fait pour éliminer les résistances aux antibiotiques. Dans la perspective d'une commercialisation, de telles constructions ne pourraient être envisagées" (avis du 28/3/1991, dossier 90.12.06).

Alors que l'Inra est pris par la « fièvre biotech » dans la deuxième moitié des années 1980, c'est la CGB qui doit refroidir I'enthousiasme de I'institut de recherche en le rappelant à la prudence : deux ou trois avis négatifs seront ainsi émis sur des expérimentations proposées par I'Inra, jugées quelque peu aventureuses. C'est le cas par exemple de la bactérie Clavibacter modifiée avec un gène codant pour une endotoxine du Bacillus thurigiensis en 1988, ou encore pour un colza avec une protéine de noix du Brésil proposé par I'Inra de Rennes en 1990. Ces exigences de caractérisation de la CGB génèrent des connaissances, les industriels étant de fait conduits à conduire des analyses, à séquencer, à chercher des détails sur les bordures, à produire des connaissances nouvelles. Par exemple on découvre que des gènes de résistance aux antibiotiques bactériens, bien qu'à l'extérieur des bandes $\mathrm{Ti}$, se retrouvent dans certaines plantes transgéniques comme le maïs Bt 176, et I'on observe des événements de 
transformation bien plus complexes que ce que I'on pouvait imaginer au départ.

\section{Les rapports avec les arènes publiques}

À cette époque, l'expertise est omniprésente au point de contenir la décision, tout en restant largement invisible dans l'espace public. Cette inexistence ou faible existence des OGM comme problème public avant 1996 s'explique certes par un travail d'enclavement du dossier par les spécialistes mais aussi par une mobilisation encore faible des associations environnementales jusqu'en 1996 et un intérêt encore fort limité des médias pour le travail de la CGB malgré les conférences de presse organisées annuellement la couverture... il faudra attendre les blocages de Greenpeace et la une «Alerte au soja fou » de Libération en novembre 1996 pour que cette situation bascule.

\section{Les inflexions de la période 1992-1996}

\section{Une formalisation du rapport à la décision}

Mais avant que la controverse publique ne prenne son envol fin 1996, des inflexions se font sentir dans et autour de la CGB à partir de 1992. Avec la loi de juillet 1992, la Commission acquiert un statut plus officiel et prend place dans un dispositif réglementaire et européen, avec une évaluation obligatoire, et de tutelle. Le parcours, entre l'avis et la décision, se formalise.

\section{La course à la mise sur le marché}

Dans le même temps, le nombre de dossiers connaît un «boom", les industriels piaffent d'impatience de voir leurs produits de première génération mis sur le marché. La concurrence est dure entre firmes comme entre États membres, qui se livrent une sorte de "course de vitesse » pour mettre sur le marché les cultures transgéniques. Et comme il n'y a pas encore de mobilisation publique en France, certaines exigences de la CGB vont être revues à la baisse par rapport à ce qu'elles étaient dans ses premières années. Ainsi, on n'exclura plus la commercialisation de plantes tolérantes aux herbicides, ou bien dans lesquelles on retrouve des gènes marqueurs de résistance aux antibiotiques, ou encore on finit par se convaincre que des constructions génétiques moins élégantes et propres qu'initialement exigé semblent cependant mériter un feu vert pour la commercialisation.

Les limites du cadrage moléculaire et de l'approche étape par étape, produit par produit

À contre-courant de ces tendances « dérégulatrices » (qui l'emportent alors aux États-Unis), un certain nombre d'inquiétudes se font jour, encore limitées aux cercles d'experts et de chercheurs, notamment autour des risques environnementaux et agronomiques liés à l'échappement des transgènes. La revue Molecular Ecology publie ainsi un dossier sur les flux de gènes en 1994. Les limites du cadrage des risques par le seul paradigme de la biologie moléculaire commencent à se faire jour, au moment où l'on s'approche du stade commercial notamment pour des colzas ou des betteraves, et où apparaît la question des flux de gènes vers les espèces voisines et les autres cultures.

On commence alors à questionner les limites cognitives du confinement dans les essais en partie $B$, et à s'interroger sur le fait que les approches step by step - avec une première étape d'essais où les exigences de confinement sont extrêmement fortes - n'apportent pas les connaissances qui seraient nécessaires à prédire ce qui va se passer en culture commerciale, à grande échelle. Ce constat est dressé notamment par les experts de I'OCDE, selon lesquels " field containment has meant that it is not possible to draw conclusions in relation to environmental effects other than those on immediate release site » [10]. Des questions similaires émanent du Comité technique permanent de la Sélection (CTPS), qui expertise la mise sur le marché des variétés en France. Son président estime que les pétitionnaires ont un peu trop " bétonnés» leurs essais afin d'obtenir aisément le feu vert dans les premières étapes $d u$ parcours réglementaire, mais que "l'objectif n'est pas de "faire passer" un dossier mais d'évaluer l'innocuité de la variété » [11]. C'est dans ce contexte qu'interviennent un certain nombre de négociations entre la CGB et le CTPS pour ajuster les deux types de réglementation. Cet épisode est intéressant en tant qu'épreuve par laquelle s'ajuste la place respective de la CGB et du CTPS dans le dispositif global d'expertise et de réglementation. C'est aussi dans ce contexte qu'apparaît la notion de biovigilance, moyen de concilier l'avancée vers la mise sur le marché tout en recueillant des données sur les impacts à grande échelle des cultures.

\section{Alertes et engagement de chercheurs}

Des divergences d'appréciation se font aussi jour dans la communauté scientifique. Des biologistes moléculaires comme Jean-Pierre Bourguin et Yves Chupeau à I'Inra s'inquiètent des usages de plantes tolérantes à des herbicides totaux, qui pourraient être plus facilement contournés. Ils conçoivent ces herbicides comme des biens publics qu'il faudrait gérer durablement. Pierre Guy, membre de la CGG et généticien à I'Inra, s'inquiète pour les mêmes raisons et adresse en 1996 plusieurs courriers à la CGB au moment où ces dossiers sont discutés. Le directeur scientifique du Cetiom, Antoine Messéan, lui, s'intéresse aux impacts agronomiques et non plus au cas par cas, étape par étape : il lance un programme inter-instituts avec des plateformes dont l'objet est d'étudier ces aspects. En 1995, Anne-Marie Chèvre, généticienne de I'Inra de Rennes, était impliquée dans un projet de recherche européen dirigé par PGS, entreprise alors leader européen des OGM, et est très amère de constater que dans la synthèse finale du projet de recherche ne figurent pas les données qu'elle a obtenues sur les distances plus élevées que prévues de dispersion du pollen et sur la possibilité de croisements interspécifiques entre colza et ses espèces cousines présentes dans nos campagnes. Elle interpelle la Direction de I'Inra sur les problèmes de l'indépendance de l'expertise et le fait qu'il est difficile de mener un certain nombre de recherches sur les risques lorsque les coordonnateurs de tels projets européens sont des entreprises. La question des conflits d'intérêt commence donc à se poser, et sera ensuite formalisée au sein de la CGB les années suivantes. Quant à certains biologistes des populations et chercheurs en écologie comme l'équipe de Pierre-Henri Gouyon ou Henri Darmency, ne s'estimant pas entendus par la CGB, ils choisissent de signer mi-1996 une pétition de chercheurs pour un moratoire de cinq ans.

\section{Réponses de la CGB}

Dès avant novembre 1996 et l'explosion de la controverse publique dans les médias, la CGB est donc confrontée à et traversée par un certain nombre de critiques et de réserves. Le président $A$. Kahn sent monter la contestation et tente de piloter par vent de face. La CGB freine alors en 1996 la plupart des dossiers d'autorisation commerciale de colza et de betterave, les plus problématiques en termes d'échappement des transgènes vers des populations naturelles, pour soutenir les dossiers de mais qui n'a pas de parent sauvage en Europe. La CGB rend notamment un avis négatif en novembre 1996 sur un essai CTPS de colza.

\section{Trois paradigmes face aux risques agro- environnementaux des OGM}

En ce milieu des années 1990, la biologie moléculaire n'est plus la seule école de recherche par laquelle on peut problématiser les risques des OGM. En simplifiant, on peut considérer trois paradigmes en présence et en concurrence (tableau 1):

- Celui de la biologie moléculaire que nous avons évoqué. 
Tableau 1. Trois cultures épistémiques face aux impacts agri-environnementaux des plantes transgéniques (d'après [5]).

\begin{tabular}{|c|c|c|c|}
\hline & $\begin{array}{l}\text { Génétique moléculaire } \\
\text { (autour de 1990, avant la } \\
\text { génomique fonctionnelle) }\end{array}$ & Biologie des populations & $\begin{array}{c}\text { Agronomie } \\
\text { (approche systèmes de culture) }\end{array}$ \\
\hline Objet d'étude & $\begin{array}{l}\text { Gènes (comme programmes, } \\
\text { molécules informationnelles) : action et } \\
\text { régulation }\end{array}$ & $\begin{array}{l}\text { Interactions dynamiques entre } \\
\text { organismes dans des écosystèmes } \\
\text { Les gènes : en flux dans des } \\
\text { métapopulations, entre compartiments } \\
\text { sauvage et cultivé }\end{array}$ & $\begin{array}{l}\text { Intervention des processus biologiques, } \\
\text { pédoclimatiques et des « pratiques } \\
\text { culturales » dans l'élaboration des } \\
\text { caractères d'un couvert végétal cultivé } \\
\text { (rendement, mais aussi qualité, } \\
\text { impacts sur l'érosion, la qualité des } \\
\text { eaux, etc.) }\end{array}$ \\
\hline Hypothèses-clés & $\begin{array}{l}\text { Héritage du dogme central « un gène- } \\
\text { une enzyme » (Crick, 1958) } \\
\text { Le comportement d'un organisme se } \\
\text { déduit essentiellement de son } \\
\text { programme génétique et s'analyse au } \\
\text { niveau moléculaire }\end{array}$ & $\begin{array}{l}\text { Le comportement des organismes est le } \\
\text { produit évolutif d'interactions avec } \\
\text { l'environnement }\end{array}$ & $\begin{array}{l}\text { Le rendement (ou tout autre caractère } \\
\text { agri-environnemental) résulte d'une } \\
\text { interaction entre : } \\
\text { - les caractères génétiques; } \\
\text { - les conditions de milieu ; } \\
\text { - les opérations culturales }\end{array}$ \\
\hline Échelle spatiale & $\begin{array}{l}\text { Infracellulaire, espace expérimental du } \\
\text { labo }\end{array}$ & Écosystème, métapopulation, paysage & $\begin{array}{l}\text { Parcelle, exploitation, } \\
\text { bassin de production }\end{array}$ \\
\hline Échelle temporelle & Restreinte : le temps du laboratoire & $\begin{array}{l}\text { Le temps des simulations (plusieurs } \\
\text { décennies) voire de l'évolution }\end{array}$ & $\begin{array}{l}\text { Le temps de l'exploitation (rotations) et } \\
\text { des simulations (plusieurs décennies } \\
\text { dans GeneSys) }\end{array}$ \\
\hline Mode de connaissance & Instrumentation, manipulation & Terrain, statistiques, modélisation & $\begin{array}{l}\text { Expérimentation au champ, } \\
\text { modélisation }\end{array}$ \\
\hline $\begin{array}{l}\text { Outils et } \\
\text { techniques }\end{array}$ & $\begin{array}{l}\text { Ultracentrifugation, PCR, } \\
\text { Western/Southern blot, transgenèse }\end{array}$ & $\begin{array}{l}\text { Mathématique, statistiques, } \\
\text { modélisation }\end{array}$ & $\begin{array}{l}\text { Expérimentation pluriannuelle, } \\
\text { statistiques, enquête, modélisation }\end{array}$ \\
\hline OGM : de nouveaux risques? & $\begin{array}{l}\text { NON : familiarité : «phenotypes are } \\
\text { determined by the expression of genes in } \\
\text { the organisms, not by the method by } \\
\text { which they have been introduced » } \\
\text { (Miller et al., 1993) } \\
\text { Les OGM : plus prédictibles et plus sûrs }\end{array}$ & $\begin{array}{l}\text { OUI? } \\
\text { Nouvelles incertitudes liées à la } \\
\text { dissémination des transgènes et à leurs } \\
\text { impacts sur les écosystèmes }\end{array}$ & $\begin{array}{l}\text { OUI? } \\
\text { Impacts indirects et cumulatifs sur les } \\
\text { pratiques agricoles? }\end{array}$ \\
\hline $\begin{array}{l}\text { Où est } \\
\text { le problème? }\end{array}$ & $\begin{array}{l}\text { Dans la construction (ex : séquence } \\
\text { codant pour des proteines } \\
\text { « indésirables »; recombinaison virale, } \\
\text { etc.) }\end{array}$ & $\begin{array}{l}\text { - Effets écologiques de la dispersion } \\
\text { d'un nouveau trait génétique? } \\
\text { - Impacts sur la biodiversité ? }\end{array}$ & $\begin{array}{l}\text { - Durabilité des innovations? } \\
\text { - Changement induits des pratiques? } \\
\text { - Coexistence OGM/non-OGM et } \\
\text { maintien d'une pluralité } \\
\text { d'agricultures? }\end{array}$ \\
\hline La solution & $\begin{array}{l}\text { - Propreté et stabilité de la construction } \\
\text { génétique ; } \\
\text { - Maîtrise du site d'insertion et de } \\
\text { l'expression ; } \\
\text { - Confinement biologique (gene use } \\
\text { restriction technology) }\end{array}$ & \multicolumn{2}{|c|}{$\begin{array}{l}\text { Nécessaire biovigilance à grande échelle } \\
\text { Pouvoir prévoir et gérer (pas toujours supprimer)... }\end{array}$} \\
\hline $\begin{array}{l}\text { Enjeux cognitifs et projets } \\
\text { intellectuels sous-jacents } \\
\text { à l'implication dans les } \\
\text { recherches sur les risques }\end{array}$ & $\begin{array}{l}\text { - Perfectionnement des techniques de } \\
\text { transgenèse ; } \\
\text { - Étude des mécanismes fondamentaux } \\
\text { de l'expression et la régulation des } \\
\text { gènes (silencing, etc.) et de } \\
\text { l'organisation du génome } \\
\text { (transpositions, fluidité, etc.) }\end{array}$ & $\begin{array}{l}\text { Approfondir les théories de biologie } \\
\text { évolutive (métapopulations, etc.) et } \\
\text { viser une écologie prédictive }\end{array}$ & $\begin{array}{l}\text { - Modéliser les interactions : génome x } \\
\text { environnement x pratiques culturales; } \\
\text { - Évaluation ex ante de caractéristiques } \\
\text { variétales, en amont des programmes } \\
\text { de sélection; } \\
\text { - Outils systémiques d'aide à la } \\
\text { décision pour une agriculture durable } \\
\text { et une gouvernance de l'agriculture à } \\
\text { l'échelle de territoires }\end{array}$ \\
\hline
\end{tabular}

- Celui de la biologie des populations. PierreHenri Gouyon souligne ainsi que le gène n'est pas seulement une molécule programme, mais quelque chose qui est en flux dans des métapopulations, que l'on doit chercher à modéliser sur des périodes et de longues durées. Selon lui, « on entend dire qu'à partir du moment où l'on ne change qu'un seul gène connu, il est très facile de prévoir les modifications induites : c'est complètement faux, changer un gène peut modifier beaucoup d'éléments dans l'écologie d'une population. [12]» II compare l'évaluation de la sécurité moléculaire à la CGB au test mécanique de bon fonctionnement d'une voiture, mais estime qu'une expertise plus systémique est nécessaire, comparable au « code de la route ».
- Celui de l'agronomie systémique, enfin. Cette approche repose notamment sur des projets de recherche concernant les impacts agronomiques dans une tradition de modélisation des systèmes de culture et une modélisation des flux de gènes pour gérer la coexistence des cultures, avec les travaux de J.-M. Meynard, A. Messéan, N. Colbach, etc. 


\section{La CGB dans le tourbillon de la controverse publique}

\author{
Un environnement incertain \\ pour la CGB qui perd la maitrise \\ du dossier
}

Avec la couverture médiatique des blocages de Greenpeace en octobre 1996, puis la décision Juppé-Vasseur-Lepage de février 1997, les médias, les ONG et les politiques s'invitent dans un processus routinier et confiné d'évaluation et de décision où la CGB était maître du jeu. La "décision Juppé » marque un coup $d^{\prime}$ arrêt à la règle implicite de l'avis conforme aux décisions et conduit à la démission d'Axel Kahn. Suit jusqu'en novembre 1997 une incertitude sur la nouvelle majorité parlementaire puis sur sa politique en matière d'OGM. C'est ensuite la préparation de la Conférence de citoyens de juin 1998, les incertitudes sur le renouvellement des membres de la CGB, le recours de Greenpeace au Conseil d'État, l'avancée vers le moratoire européen... Bref, toute une séquence d'événements distendent les liens entre la gestion politique du dossier OGM et la CGB, qui n'est plus le centre $d^{\prime}$ 'impulsion du système d'action publique sur les plantes transgéniques qu'elle était autrefois.

\section{La nouvelle CGB de 1998}

A la suite de la Conférence citoyenne en 1998, une nouvelle commission est formée, avec une plus nette séparation entre gestion et évaluation des risques, dans un contexte plus large de transformation de l'expertise en réponse aux crises sanitaires des années 1990 (Sida, amiante et ESB principalement). L'heure n'est plus au modèle du comité auprès d'un ministère, enclavé dans l'appareil administratif : le modèle des agences devient la norme. C'est la mise en œuvre de principes qui se généralisent en Europe :

- L'expertise contradictoire. La nouvelle CGB reflète ainsi un triple pluralisme: place plus importante aux représentants de la société civile, diversification des disciplines représentées et nomination de membres s'étant positionné de façon critique sur la première génération d'OGM.

- La validation des avis par vote, plutôt que par consensus, avec consignation d'avis minoritaires.

- L'exigence d'indépendance: les déclarations d'intérêt sont mise en place.

- La transparence : avancée progressive, sous la pression des associations et du jeu de concurrence entre Afssa et CGB, vers la publication rapide des lieux et objets des essais, des avis puis des procès-verbaux.

\section{L'expertise s'émancipe \\ de l'administration et se diversifie}

Toutefois, même ainsi recomposée, la CGB n'est plus la clé de voûte du système de gouvernance des OGM. D'autres comités ont émergé, le Comité de biovigilance et surtout l'Afssa. Deux épisodes illustrent le jeu de concurrence qui naît entre ces différences instances en quête d'une crédibilité publique contestée. En juillet 2001 d'une part, la contamination de lots de maïs conventionnels par des OGM donne lieu à un avis non rendu public de la CGB puis à un avis très médiatisé de I'Afssa, qui marque alors des points vis-à-vis d'une opinion extrêmement sensibilisée. Inversement, 5 des 6 dossiers qui ont été examinés en commercialisation en 2003 ont fait l'objet $d^{\prime}$ avis divergents entre la CGB et l'Afssa, avec souvent des avis plus « précautionneux » de la part de la CGB que de cette dernière.

\section{La recherche s'émancipe de l'expertise}

Parallèlement, il se produit une autonomisation du champ de recherche sur les risques. Nombre d'institutions scientifiques financent des projets et appels d'offres. Ainsi le paysage est assez différent de celui de la première période. L'expertise est fragmentée entre plusieurs instances, en partie autonomisée de l'appareil administratif, ne pilote plus directement les recherches, et ne détermine plus la décision politique: il y a beaucoup de jeu entre les différents éléments du système d'action publique (figure 1).

\section{Affirmation d'une arène de l'expertise}

S'il y a affaiblissement du rôle autrefois central de la CGB dans la politique publique des OGM, on note par contre que, prise globalement, la fonction expertise sort renforcée des évolutions récentes. Plus nettement séparée des fonctions de recherche et des fonctions de décision, elle est mieux individualisée en tant que telle par les acteurs des autres arènes publiques. II y a eu affirmation d'une " arène ", d'un espace institutionnel, propre à l'expertise, avec ses règles, ses logiques, sa visibilité dans l'espace public. Le travail des experts dans cette arène fait désormais l'objet de règles et de procédures détaillées (et, à l'Afssa, il se voit doté de moyens administratifs importants).

\section{L'arène judiciaire : $3^{e}$ cercle de l'expertise?}

Un autre élément très important d'évolution de ces dernières années, lui aussi source d'affaiblissement de la CGB, est l'implication croissante de l'arène judiciaire, avec les recours associatifs sur la transparence ou sur la légalité des décisions intervenus depuis 1997, puis avec les procès des faucheurs à partir de 1999 [13], et enfin depuis peu avec des recours sur la plupart des autorisations d'essais du ministère, qui se font souvent en arguant d'une évaluation des risques défectueuse. Ainsi le tribunal correctionnel d'Orléans estime-t-il que « les distances entre les essais et les autres plantations préconisés par la CGB dans ses avis sont parfaitement insuffisantes à prévenir une possible contamination (...) aucune des précautions préalables imposées

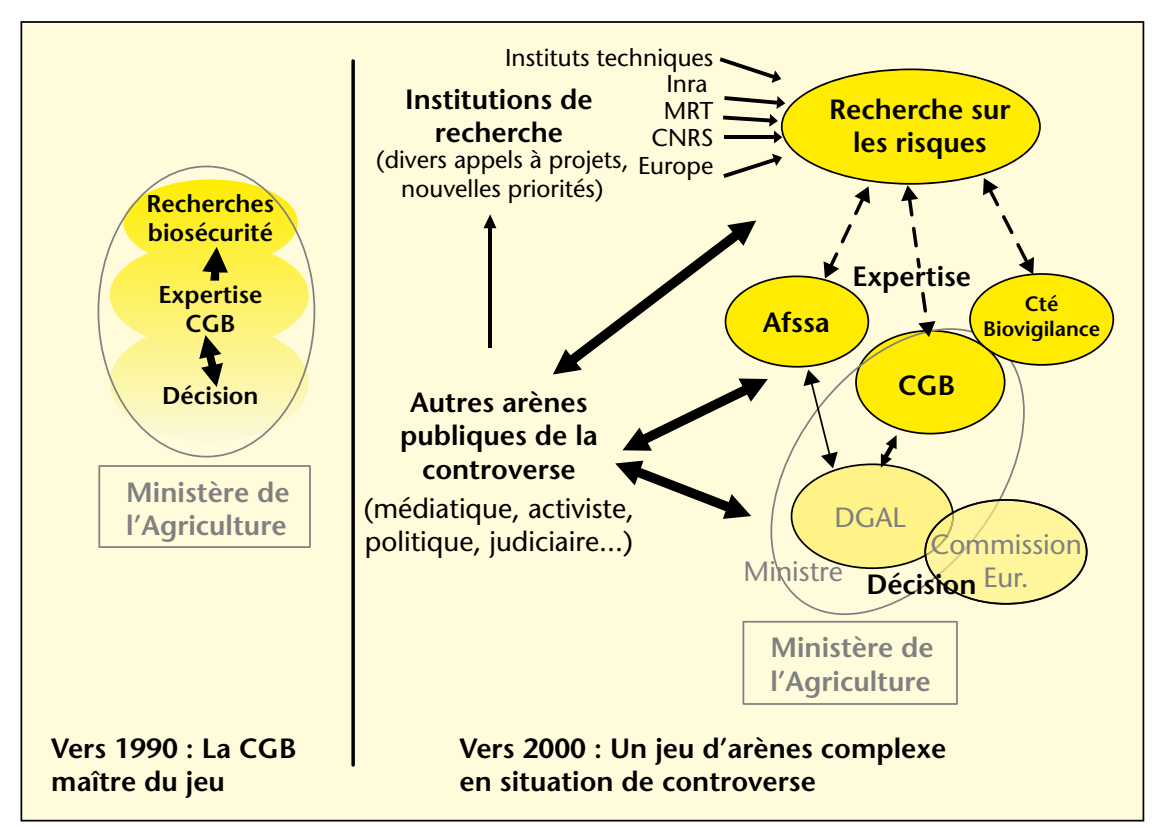

Figure 1. Le système d'action publique « expertise, décision, recherche en biosécurité » avant et après l'explosion de la controverse publique sur les OGM. 
par la CGB ne répond à la possible dissémination par transfert de gènes avec les bactéries ou les champignons dans le sol». [13] À l'inverse, le tribunal administratif de Clermont-Ferrand affirme que les avis de la CGB « sont spécialement fondés sur les circonstances que les protéines PAT, NPTII et GFP ont fait l'objet d'études approfondies n'ayant révélé aucun impact sur la santé (...) il ne saurait être contesté que le ministère de l'Agriculture a en outre assorti ses décisions (...) de mesures de précaution et de contrôle consistant notamment en l'éloignement de $400 \mathrm{~m}$ (...) ainsi qu'en la mise en place d'une bordure quadripartite de mais non transgénique et dont la patente inefficacité n'a pas été démontrée par la Confédération paysanne (...) la CGB a d'ailleurs qualifié de "très faible" le niveau de dispersion des transgènes dans l'environnement... » [14].

Si comme on le voit ces procès connaissent des issues variées, tantôt favorables, tantôt défavorables à l'administration et aux entreprises, ils ont pour effet de mettre les juges en situation de peser si les distances de sécurité avec les cultures voisines sont suffisantes, si les transferts latéraux ont été suffisamment pris en compte, si le maïs est mellifère, $s$ 'il faut ou non connaître la localisation précise d'une parcelle pour bien réaliser un travail d'évaluation à la CGB, si les lignées transgéniques sont stables... Toutes ces questions, qui auparavant étaient l'apanage $d^{\prime}$ un cercle d'experts, se retrouvent portées devant la justice. Le travail des comités d'experts se trouve donc mis à l'épreuve dans l'arène judiciaire. On parle souvent du $2^{\mathrm{e}}$ cercle de l'expertise, celui de représentants de la société et des sciences économiques et sociales qui évalueraient les enjeux sociétaux à côté du premier cercle qui se limite aux risques biologiques. Mais il nous semble voir poindre ici un " troisième cercle », celui de l'évaluation judiciaire du travail des instances d'expertise.

\section{Conclusion}

Les décideurs et industriels peuvent avoir le sentiment que la configuration actuelle du dis- positif d'action publique (avec une expertise fragmentée où les divergences entre comités sont visibles sur la place publique donnant des prises aux opposants et aux juges) n'est pas de nature à contribuer à clore la controverse publique.

La tentation est alors forte de reconcentrer I'expertise des OGM dans une instance unique, centralisée et de durcir et réenclaver l'expertise pour la protéger des arènes activiste, médiatique et judiciaire.

C'est une option de ce type qui fut adoptée par la commission en centralisant l'expertise au sein de l'Efsa pour limiter le jeu des divergences d'évaluation entre États membres. Cette tentation « reconcentratrice» se ressent dans le " projet de loi relatif aux organismes génétiquement modifiés » voté au Sénat en mars 2006, qui prévoit la création d'un " Haut Conseil des Biotechnologies » vertical, regroupant les activités d'évaluation des OGM de la CGB et du Comité de Biovigilance...

D'un autre côté, avec les recours sur les autorisations d'essais, les mouvements des maires, les régions sans OGM, il apparaît une nouvelle et forte aspiration de toute une série d'acteurs sociétaux à une décentralisation de la gouvernance des OGM (et de l'agriculture en général). Certains experts eux-mêmes penchent d'ailleurs également pour une certaine dose de décentralisation de l'expertise sur les impacts des OGM, notamment à propos des conditions de mise en culture et des règles de coexistence. Ils pensent en effet que cela permettrait d'ouvrir, région par région, de nouveaux espaces de négociation et de compromis entre les acteurs, un peu comme le proposait M. Pelsy.

L'arbitrage entre ces deux types d'options, recentralisatrice ou décentralisatrice, dont nous avons un peu forcé les traits pour mieux faire saisir les deux pôles d'un spectre de possibles, sera pour l'expertise I'un des enjeux importants de la future loi sur les OGM. [15].

\section{RÉFÉRENCES}

1. JOLY PB, ASSOULINE G, KRÉZIAK D, LEMARIÉ, MARRIS C, ROY A. L'Innovation controversée : le débat public sur les OGM en France, Rapport de recherche à la DGAL, 2000.

2. ROY A. Les experts face au risque: le cas des plantes transgéniques. Paris : PUF, 2001.

3. GRANJOU C. La gestion du risque entre technique et politique. Comités d'experts et dispositifs de traçabilité à travers les exemples de la vache folle et des OGM, Thèse Univ. R. Descartes, 2004.

4. MARRIS C, RONDA S, JOLY PB, BONNEUIL C. How the French GM controversy led to the reciprocal emancipation of scientific expertise and policy making. Sci Public Policy 2006; 32(4) : 301-8.

5. BONNEUIL C. Cultures épistémiques et engagement des chercheurs dans la controverse OGM. Natures Sciences Société $2006 ; 14(3)$ : 257-68.

6. DESHAYES A. Evaluation des conséquences des choix scientifiques et technologiques dans le domaine des applications des biotechnologies à l'agriculture et à l'industrie agro-alimentaire. Rapport pour I'OPECST, sept 1990.

7. OCDE. Le « livre bleu » de l'OCDE, 1986.

8. KAHN A. La Commission du Cénie. Biomoléculaire. Son rôle, son action et ses buts. Déc. 1990 ; (p. 6).

9. KAHN A. P.-V. de la séance du 7 oct. 1988.

10. OECD. Field releases of transgenic plants, 1986 1992. An analysis. Paris, 1993 ; (p. 7).

11. MARROU J. Note. Président du CTPS, 1993. Fonds Marrou récemment versé aux Archives Nationales.

12. GOUYON PH. Les Harmonies de la nature à l'épreuve de la biologie : évolution et biodiversité. Paris : Inra Éditions, 2001 ; (p. 29).

13. BONNEUIL $C$, JOLY PB, MARRIS C. Disentrenching experiment? The construction of $\mathrm{GM}$-crop field trials as a social problem in France. Science, Technol Human Values 2008 : 33 ; (à paraître).

14. TC Orléans, 27 juin 06.

15. TA Clermont-Ferrand 4 août 2006. 